\title{
Sustained impact of subcutaneous immunotherapy among patients with allergic rhinitis who experienced treatment delay due to the COVID-19 pandemic: A multicenter, two-arm, real-world study
} \author{
Qianhui Qiu ${ }^{7}$ \\ ${ }^{1}$ Zhujiang Hospital, Southern Medical University \\ 2 the Third People's Hospital of Changzhou \\ ${ }^{3}$ First People's Hospital of Foshan \\ ${ }^{4}$ People's Hospital of Guangxi Zhuang Autonomous Region \\ ${ }^{5}$ Liuzhou people's Hospital \\ ${ }^{6}$ Yong Loo Lin School of Medicine, National University of Singapore \\ ${ }^{7}$ Zhujiang Hospital, Southern Medical University, Guangzhou, China
}

SUIZI ZHOU ${ }^{1}$, Yibin Liu ${ }^{1}$, Jianrong Xue ${ }^{2}$, Juan Tang ${ }^{3}$, Qingqing $\mathrm{Yu}^{3}$, Shenhong $\mathrm{Qu}^{4}$, Shaojie Zhang ${ }^{4}$, Binyu Mo ${ }^{5}$, Jihui $\mathrm{Li}^{5}$, Yinhong Liu ${ }^{1}$, Yueying Yang ${ }^{1}$, De Yun Wang ${ }^{6}$, and

May 31, 2021

\begin{abstract}
Background: The aim of this study is to investigate the impact of COVID-19 related treatment delay on subcutaneous immunotherapy (SCIT) efficacy in patients with allergic rhinitis (AR). Methods: The study was performed in 643 patients with SCIT appointments between February 1 and May 31, 2020. The clinical assessment, performed at baseline (V0) and one year later (V1), included visual analogue scale (VAS); daily symptom score (dSS); daily medication score (dMS); combined symptom and medication scores (CSMS); quality of life (QoL); self-rating anxiety scale (SAS); and self-rating depression scale (SDS) for each patient. Results: At V0, 249 patients were treated on schedule, and 394 were delayed ( \pm 4.68 weeks). Among them, 319 patients (105 on schedule, and 214 delayed) also completed the assessments at V1, with the absence of $25.39 \%$ patients due to completion of SCIT, and $25.35 \%$ patients were withdrawal. The results of all assessments were within the normal range for all patients at V0 and V1, with the exception of a slightly higher SDS score (56.13) at V0. In the SCIT delayed group. there was a significant positive correlation between the length (weeks) of the delay and SDS score, and this was significantly higher in patients with poor control of nasal symptoms. Conclusions: This study showed the long-term efficacy of SCIT for AR patients, including those who have had to delay normal therapy due to the COVID-19 outbreak. The psychological status of SCIT patients in response to lockdown of hospital services during this critical period should be considered.
\end{abstract}

Title page

Sustained impact of subcutaneous immunotherapy among patients with allergic rhinitis who experienced treatment delay due to the COVID-19 pandemic: A multicenter, two-arm, realworld study

Running title: SCIT delayed in patients with AR during COVID-19

Suizi Zhou ${ }^{\mathrm{a}, \mathrm{b}^{*}}$, Yibin Liu ${ }^{\mathrm{a}, \mathrm{b}^{*}}$, Jianrong Xue ${ }^{\mathrm{c},{ }^{*}}$, Jun Tangd, ${ }^{\mathrm{d}, *}$ Qingqing $\mathrm{Yu}^{\mathrm{d}, *}$, Shenhong $\mathrm{Qu}^{\mathrm{e},{ }^{*}}$, Shaojie Zhang $^{\mathrm{e}, *}$, Binyu Mo ${ }^{\mathrm{f},}$, Jihui $\mathrm{Li}^{\mathrm{f}, *}$, Yinhong Liu ${ }^{\mathrm{a}}$, Yueying Yang, ${ }^{\mathrm{a}, \mathrm{b}}$, De-Yun Wang, ${ }^{\mathrm{g}}$, Qianhui Qiu ${ }^{\mathrm{h}, \mathrm{b}}$ 
aDepartment of Otolaryngology, Zhujiang Hospital, Southern Medical University, Guangzhou, Guangdong Province, China;

bThe Second School of Clinical Medicine, Southern Medical University, Guangzhou, Guangdong Province, China;

'Department of Otorhinolaryngology, the Third People's Hospital of Changzhou, Changzhou, Jiangsu Province, China;

dDepartment of Otorhinolaryngology, The First People's Hospital of Foshan, Foshan, Guangdong Province, China;

eDepartment of Otorhinolaryngology, People's Hospital of Guangxi Zhuang Autonomous Region, Nanning, Guangxi Province, China;

${ }^{\mathrm{f}}$ Department of Otorhinolaryngology, Liuzhou people's Hospital, Liuzhou, Guangxi Province, China;

gDepartment of Otolaryngology, National University of Singapore, National University Health System, Singapore, Singapore;

h Department of Otolaryngology, Guangdong Provincial People's Hospital and Guangdong Academy of Medical Sciences, Guangzhou, Guangdong Province, China.

${ }^{*}$ These authors contributed equally to this work.

Corresponding author: Qianhui Qiu, E-mail:

qiuqianhui@hotmail.com;

De-Yun Wang, E-mail: entwdy@nus.edu.sg

Funding statement

This study was supported by grants from National Nature Science Foundation of China No.81873690 (to Q-QH).

Words: 3500

\section{Abstract}

Background: The aim of this study is to investigate the impact of COVID-19 related treatment delay on subcutaneous immunotherapy (SCIT) efficacy in patients with allergic rhinitis (AR).

Methods: The study was performed in 643 patients with SCIT appointments between February 1 and May 31, 2020. The clinical assessment, performed at baseline (V0) and one year later (V1), included visual analogue scale (VAS); daily symptom score (dSS); daily medication score (dMS); combined symptom and medication scores (CSMS); quality of life (QoL); self-rating anxiety scale (SAS); and self-rating depression scale (SDS) for each patient.

Results: At V0, 249 patients were treated on schedule, and 394 were delayed ( 74.68 weeks). Among them, 319 patients (105 on schedule, and 214 delayed) also completed the assessments at V1, with the absence of $25.39 \%$ patients due to completion of SCIT, and $25.35 \%$ patients were withdrawal. The results of all assessments were within the normal range for all patients at V0 and V1, with the exception of a slightly higher SDS score (56.13) at V0. In the SCIT delayed group. there was a significant positive correlation between the length (weeks) of the delay and SDS score, and this was significantly higher in patients with poor control of nasal symptoms.

Conclusions: This study showed the long-term efficacy of SCIT for AR patients, including those who have had to delay normal therapy due to the COVID-19 outbreak. The psychological status of SCIT patients in response to lockdown of hospital services during this critical period should be considered. 
Key words: COVID-19, clinical manifestation, delayed therapy, depression, subcutaneous immunotherapy (SCIT)

\section{Abbreviations used}

AR: allergic rhinitis

AIT: allergen-specific immunotherapy

IgE: immunoglobulin $\mathrm{E}$

COVID-19: coronavirus disease 2019

SCIT: subcutaneous immunotherapy

RWE: real world evidence

WAO: World Allergy Organization

EAACI: European Academy of Allergy and Clinical Immunology

ARIA: Allergic Rhinitis and Its Impact on Asthma

VAS: visual analogue scale

dSS: daily symptom score

dMS: daily medication score

CSMS: combined symptom and medication scores

QoL: quality of Life

RQLQ: Rhinoconjunctivitis Quality of Life Questionnaire

SAS: self-rating anxiety scale

SDS: self-rating depression scale

JACI: Journal of Allergy and Clinical Immunology

Dp: dermatophagoides pteronyssinus

Df: dermatophagoides farinae

SD: standard deviation

CRS: chronic rhinosinusitis

CHD: coronary heart disease

FD: functional dyspepsia

PI: primary insomnia

RT: radiotherapy

H1A: oral and/or topical (eyes or nose) non-sedative H1 antihistamines

INS: intranasal corticosteroids

IgG4: immunoglobulin G4

IgE: immunoglobulin E

\section{Introduction}


The prevalence of allergies is increasing, and the most common manifestation of this are the respiratory allergic diseases such as allergic rhinitis (AR) and asthma ${ }^{1,2}$. Aomg patients with AR and allergic asthma, allergen-specific immunotherapy (AIT) is the only treatment option that induces clinical and immunologic allergen-specific immune tolerance, thus conferring a long-lasting clinical benefit ${ }^{3,4}$. Subcutaneous immunotherapy (SCIT), which is the most common form of AIT, has been demonstrated to be highly effective for treating patients with a mite allergy, and/or seasonal pollinosis ${ }^{5}$. SCIT usually involves administering a gradually increasing dose of the specific allergen to allergic patients until the effective dose is reached, which takes several weeks and is followed by two or more years of maintenance doses ${ }^{6}$. Patient compliance and education during treatment has been shown to play an important role in guaranteeing the disease-modifying effect of SCIT ${ }^{7}$. However, the impact of treatment delays on the condition of SCIT patients with allergic diseases has not yet been reported.

At the end of 2019, the global community witnessed the outbreak of the coronavirus disease 2019 (COVID19) in China, which has subsequently had a tremendous influence on clinical, educational, research, and community responsibilities around the world ${ }^{8}$. In the event of such a global infectious pandemic, allergy and immunology specialists have suggested that rapid and drastic measures may be needed, which limit or require adjustment of ambulatory allergy services ${ }^{9}$. Affected by the pandemic, many patients who were receiving SCIT were forced to discontinue or postpone treatment in the hospitals, which led us to consider whether these patients experienced any physical and/or mental impacts because of treatment delay during this period. Real world evidence (RWE) allows for estimates of effectiveness rather than efficacy in various typical practice settings, as well as the examination of clinical outcomes in a diverse study population, which is representative of patients observed in clinical practices ${ }^{5}$. In this research, we follow up on the physical and mental outcomes on SCIT patients that experienced treatment delays, based on RWE up to one year. This will enable us to develop novel strategies for future SCIT management during the COVID-19 pandemic.

According to the recommendations of World Allergy Organization (WAO) and European Academy of Allergy and Clinical Immunology (EAACI $)^{10,11}$, a comprehensive set of end-points were chosen for our research. To measure symptom and medication (1) these included visual analogue scale (VAS); daily symptom score (dSS); daily medication score (dMS); and combined symptom and medication scores (CSMS). To measure quality of life (QoL) (2), and psychological burden (3) these included self-rating anxiety scale (SAS); and self-rating depression scale (SDS). These commonly used indices were selected to demonstrate the severity of symptoms, use of medication, quality of life, effect of anxiety and depression related symptoms in patients with delayed SCIT during the COVID-19 outbreak.

\section{Methods}

\section{Study design}

This multicenter, two-armed, real-world study included patients with AR and IgE-mediated sensitization to dermatophagoides pteronyssinus $(\mathrm{Dp})$ and/or dermatophagoides farinae (Df). Patients were undergoing ongoing SCIT with a treatment visit during the period between February 1 and May 31, 2020 (during the COVID-19 outbreak in China) for the first visit (V0) and for follow-up 1-year later (V1). They were enrolled from the departments of otorhinolaryngology from five tertiary hospitals (in three provinces in southern China), including Zhujiang Hospital of Southern Medical University, the Third People' s Hospital of Changzhou, the First People's Hospital of Foshan, the People's Hospital of Guangxi Zhuang Autonomous Region and Liuzhou People's Hospital. Approval to conduct this study was obtained from the institutional review board of all participating hospitals. The patients in this study were all from hospitals not designated for COVID-19 infected patients. Special measures were performed to prevent patients with a COVID-19 infection from entering the hospitals. The patients received at least one reminder call a week prior to their SCIT appointment.

A standard protocol for SCIT @ (50\% Dp and 50\% Df, Allergopharma Joachim Ganzer KG, Reinbek, Germany) was used for all patients in both the build-up and maintenance phases. For the build-up phase, SCIT\& dose was gradually increased every 1-2 weeks until the maximum tolerated dose for the individual 
was reached, and the treatment for a total of 18 weeks including the dose increases.. In the build-up phase, injections at an interval of more than 2 weeks were regarded as delayed treatment. For the maintenance phase, the maximum tolerated dose was given subcutaneously every 4-6 weeks for at least 2 years. During the maintenance phase, injections at an interval of more than 6 weeks were regarded as a delayed treatment. The dosage adjustment plan in the case of delayed treatment for SCIT patients in the build-up phase was as follows: (1) > 2 weeks, the dose was adjusted to $50 \%$ of the last dose; $>4$ weeks, start again from the initial dose concentration. The dosage adjustment plan in the case of delayed treatment for SCIT patients in the maintenance phase was: $(1)>6$ to 8 weeks, the dose was adjusted to $50 \%$ of the last dose; (2) $>8$ weeks, the dose was adjusted to $5 \%$ of the last dose; $(3)>52$ weeks, and the treatment was resumed from the starting concentration. The course of SCIT in this study was at least 3 years.

\section{Study patients}

Patients who met the following inclusion criteria were included in the study: (1) a positive allergy test result to Dp and Df by either skin prick test [scored ++ or above (Alutard, ALK-Abellórd, Denmark)] or by PharMacia CAP system for serum sIgE level [?] 2 (LG Chem, South Korea); (2) a confirmed diagnosis of AR based on the criteria of 'Allergic Rhinitis and Its Impact on Asthma (ARIA)'12; (3) with ongoing SCIT in one of the selected hospitals before the COVID-19 outbreak; (4) with signed informed consent for participating in this study.

Exclusion criteria were as follows: (1) forced expiratory volume in 1 second (FEV1) $<80 \%$, as predictive value in patients with allergic asthma; (2) pregnant or lactating women; and (3) patients with other contraindications to SCIT.

\section{Assessment of clinical manifestations}

The data were collected by paper- or web-based questionnaires for both V0 and V1. Children (aged under 14) completed the questionnaires with the help of their parents. The clinical manifestations assessed were symptoms $\left(\mathrm{VAS}^{13,14}, \mathrm{dSS}^{15}\right)$, medication $\left(\mathrm{dMS}^{16}, \mathrm{CSMS}^{17}\right)$, quality of life $\left(\mathrm{QoL}^{18,19}\right)$, psychological burdens (SAS, SDS ${ }^{20,21}$ ) and clinical control of SCIT patients in the week prior to treatment (see details in supplement ).

\section{Statistical analysis}

Statistical analyses were conducted with GraphPad Prism 7. Continuous variables are presented as means and standard deviations (SD) or interquartile ranges (IQR) as appropriate, and the categorical variables are presented as counts and percentages. Demographic data and clinical characteristics were computed for all included participants and compared using chi-square or Fisher exact tests. Differences in clinical outcomes were compared using nonparametric Mann-Whitney tests or Kruskal-Wallis test, where appropriate. The correlation of treatment delay time interval and the score of SDS at V0 was evaluated by Spearman's correlation analysis. $\mathrm{P}$ value of $<0.05$ was considered as statistically significant.

\section{Results}

\section{Epidemiologic and demographic characteristics}

Of the 654 patients who were assessed for eligibility, 11 were excluded as their last injection date of SCIT was not recorded in the questionnaire. At V0, 249 patients $(38.72 \%)$ were receiving SCIT on schedule while 394 patients $(61.28 \%)$ were receiving postponed SCIT. At V1, 161 patients $(25.04 \%)$ had already completed SCIT (for more than three years), and 163 patients $(25.35 \%$ ) had withdrawn from SCIT. Thus, a total of 105 patients treated on schedule $(32.92 \%)$ and 214 patients with treatment delay $(67.08 \%)$ were available for follow-up in V1. Of these, a few patients were excluded due to missing data or inappropriately filled in questionnaires (Figure 1 ).

The median time interval of delayed SCIT was 7 weeks, and ranged from 1 to 30 weeks. The groups of scheduled SCIT and delayed SCIT were comparable in terms of the treatment phases and diagnosis. There was no significant difference for age, gender, and adverse reactions between the scheduled SCIT and delayed 
SCIT groups at both V0 and V1. None of the study patients were infected by COVID-19 during the study (Table 1 ).

\section{Clinical efficacy in patients with delayed SCIT compared to on-schedule SCIT}

For subjective symptoms, the mean +- SD value of VAS was $2.67+-2.10$ at V0 and $2.271+-1.53$ at V1 for patients with delayed SCIT, whereas patients with scheduled SCIT had a higher score of $3.08+-2.13$ at V0 $(\mathrm{P}=0.0191)$ and $2.30+-1.82$ at V1 (Figure 2, $\mathbf{A})$. The number of patients with delayed SCIT who did not feel any symptoms was $14.79 \%$, and patients who reported moderate symptoms was $21.01 \%$. This was more than $4.27 \%(\mathrm{P}=0.0001)$, and less than $30.33 \%(\mathrm{P}=0.0136)$ than in patients with scheduled SCIT at V0. Severe symptoms were reported in $0.47 \%$ of patients with delayed SCIT, which was less than $3.84 \%$ than in patients with scheduled SCIT $(\mathrm{P}=0.0424)$ (Table 2$)$.

With a similar tendency as VAS, the mean +- SD value of dSS was $0.63+-0.50$ in patients with delayed SCIT, which was also lower than the scheduled SCIT group at V0 $(0.76+-0.60, \mathrm{P}=0.0208)$. However, the score of $0.60+-0.43$ in patients with delayed SCIT was significantly higher than the score of $0.51+-0.47$ for scheduled SCIT patients at V1 $(\mathrm{P}=0.0375)$ (Figure 2, B ). The conjunctival symptom of watery eyes at V0, and nasal symptoms of itchy nose and of sneezing at V1, showed a significant difference between the delayed and scheduled SCIT groups $(\mathrm{P}=0.0267, \mathrm{P}=0.0001$, and $\mathrm{P}=0.0267$ respectively) (Table 2 ).

For rescue medication, medication usage among patients was significantly increased in the delayed SCIT group compared to the scheduled SCIT group $(\mathrm{P}=0.0478)$ at V0 (Figure 2, $\mathbf{C}$ ), particularly the usage of intranasal corticosteroids (INS) $(\mathrm{P}=0.0177)$. For allergic control, intake of INS with or without H1antihistamine was increased up to nearly $50 \%$ at V0 in the delayed SCIT group. However, no major difference of dMS was observed between the groups at V1 (Table 2 ). Moreover, the score of CSMS, which balances both symptoms and the need for anti-allergic medication in an equally weighted manner, shows no statistical difference in patients with delayed or scheduled SCIT at V0 and V1 (Figure 2, D ).

For QoL assessment, the mean value with upper to lower 95\% CI of QoL grade was 20.89 (18.73 to 23.05) and 26.97 (24.17 to 29.76) in patients with delayed and scheduled SCIT at V0, respectively $(\mathrm{P}<0.0001)$, showing less life damage among delayed SCIT patients. The remarkable difference in the grade of 20.71 (18.15 to 23.27 ) in patients with delayed SCIT in comparison to 17.85 (13.96 to 21.74) in patients with scheduled SCIT was seen at V1 $(\mathrm{P}=0.0334)$ (Figure 2, $\mathbf{E})$. The number of patients with activity problems $(\mathrm{P}<$ $0.0001)$ and generalized symptoms $(\mathrm{P}=0.0093)$ during the past week at V0 were found to be prominently reduced in the delayed SCIT group, while at V1, sleep problems, generalized symptoms, practical problems, nasal symptoms, ocular symptoms, and emotional problems were prominently increased in comparison with the scheduled SCIT group (all $\mathrm{P}<0.05$ ) (Table 2 ).

\section{Emotional evaluation from patients with delayed and scheduled SCIT.}

To investigate the level of anxiety and depression related symptoms in patients undergoing SCIT at V0 and V1, we compared the SAS and SDS data to healthy controls: healthy subjects outside of ${ }^{22}$ or during ${ }^{23}$ the COVID-19 outbreak; COVID-19 related individuals (front-line clinical staff ${ }^{24}$ or survivors $^{25}$ of COVID-19); patients with allergy and inflammation related diseases $\left(\mathrm{AR}^{26}\right.$, asthma ${ }^{27}$, chronic rhinosinusitis $\left.(\mathrm{CRS})^{28}\right)$; patients with chronic disorders (coronary heart disease (CHD) ${ }^{29}$, functional dyspepsia (FD) ${ }^{22}$ and primary insomnia $(\mathrm{PI})^{30}$ ); and cancer patients post radiotherapy $(\mathrm{RT})^{31}$ in China.

As shown in figure 3, A, SAS in patients with delayed SCIT at V1 was $33.79+-7.95$, which was significantly lower than $40.66+-7.61$ in the scheduled SCIT group at V0, $37.96+-5.23$ of healthy control during the COVID-19 outbreak, $45.89+-1.12$ of front-line clinical staff in the COVID-19 outbreak, $43.2+-10.2$ of COVID-19 survivors, $42.23+-14.32$ of AR patients, $40.80+-8.10$ of asthma patients, $39.40+-11.55$ of CRS patients, $43.9+-5.6$ of patients with CHD, $42.07+-8.01$ of patients with FD, $51.8+-10.8$ of patients with PI, and $55.69+-10.01$ of cancer patients post RT (all $\mathrm{P}<0.0001$ ). However, there was no significant difference between patients with delayed SCIT at V1 $(33.79+-7.95)$ when compared to patients with scheduled SCIT at V1 $(34.84+-7.07)$, patients with delayed SCIT at V0 $(34.87+-8.48)$, or to healthy 
controls outside of the COVID-19 outbreak (33.85 +- 6.4).

As shown in figure 3, B , the SDS score in delayed SCIT patients at V1 (38.51 +- 12.88) was significantly lower when compared to delayed SCIT patients at V0 $(40.48+-14.86, \mathrm{P}<0.01)$, and also scheduled SCIT patients at both V1 $(44.67+-14.74, \mathrm{P}<0.0001)$ and V0 $(56.13+-10.73, \mathrm{P}<0.0001)$. Similarly, the SDS score in delayed SCIT patients at V1 was not statistically different from the healthy controls outside of the COVID-19 outbreak (34.81 +- 7.43). However, this was dramatically decreased when compared with healthy patients during the COVID-19 outbreak (44.2 +- 5.46), front-line clinical staff in the COVID-19 outbreak (50.13 +- 1.813), COVID-19 survivors (47.3 +- 13.1), patients with AR (43.32+- 13.78), asthma (44.90 +- 9.10), CRS (54.05 +- 10.96), CHD (53.60+- 8.70), FD (43.27+- 10.04), PI (55.40+- 8.90), and cancer patients post RT $(59.05+-9.40)$, all $\mathrm{P}<0.05$. Among them, scheduled SCIT patients at V1 and V0, delayed SCIT patients at V0, COVID-19 survivors, patients with AR, asthma, CRS, CHD, FD, PI and cancer patients post RT had a SDS scores over the depression threshold of 53 .

The proportion of patients without depression from the scheduled and delayed SCIT groups were $28.11 \%$ and $66.86 \%$ at V0, and $60.95 \%$ and $82.12 \%$ at V1, respectively. The percentage of; mildly depressed patients for the scheduled and delayed groups was $60.33 \%$ and $29.33 \%$ at V0, $32.35 \%$ and $18.96 \%$ at V1; and moderately depressed patients, $11.16 \%$ and $3.81 \%$ at V0, $5.80 \%$ and $1.42 \%$ at V1, respectively (all $\mathrm{P}<0.0001$ ). Severely depressed patients accounted for $0.40 \%$ and $0.90 \%$ at V0 and V1 respectively from the scheduled SCIT group, with $0 \%$ in the delayed SCIT group at both timepoints (Figure 3, C ). In addition, a positive correlation of SDS and time interval of SCIT delay $(\mathrm{r}=0.3975, \mathrm{P}<0.0001)$ was observed (Figure 3, $\mathbf{D})$. The proportion of SAS, as well as the correlation between SAS and delayed SCIT time interval at V0 are shown in figure S1.

\section{Clinical outcomes and depressive symptoms were interlinked}

We further split the cohort into a non-depressed and depressed group based on SDS scores to determine whether psychological status had an influence on clinical manifestations. In figure $4, \mathbf{A}-\mathbf{E}$, it can be seen that for SCIT patients, the mean values of VAS (2.46 at V0; 2.10 at V1), dSS (0.57 at V0; 0.52 at V1), CSMS (at $1.0 \mathrm{~V} 0$; at $0.74 \mathrm{V1}$ ), and $\mathrm{QoL}(19.12$ at V0; 17.57 at V1) in the non-depressed group are significantly lower than VAS (3.20 at V0; 2.83 at V1), dSS (0.74 at V0; 0.72 at V1), CSMS (1.5 at V0; 0.96 at V1), QoL (25.41 at V0; 25.86 at V1) in the depressed group (all $\mathrm{P}<0.05$ ).

The mean SDS score in patients undergoing SCIT was remarkably increased in patients with poorly controlled (42.16 at V0 and 39.65 at V1) and well controlled symptoms (40.54 at V0 and 41.2 at V1), when compared to those with completely controlled symptoms (32.24 at V0 and 29.58 at V1) (P < 0.01) (Figure $\mathbf{4}, \mathbf{F}$ ). The difference between clinical manifestations and clinical control in comparison to symptoms of anxiety are shown in figure $\mathbf{S} 2$.

\section{Discussion}

In the context of developing novel future strategies for SCIT management during the COVID-19 pandemic, our research confirmed that long-term efficacy of SCIT is not negatively affected by COVID-19 related treatment delay, in patients with $\mathrm{AR}$ (some also with asthma). In addition, we also demonstrated the presence of psychological symptoms, especially depressive symptoms, in patients undergoing SCIT during the COVID-19 outbreak.

During this study, we found that $25.35 \%$ of SCIT patients had withdrawn from treatment before V1, which is similar to findings from the recent EAACI survey in which $75 \%$ of patients underwent maintenance phase SCIT during the COVID-19 pandemic ${ }^{32}$. An objective reason for this would be that social quarantine was called for in response to the COVID-19 outbreak; and a subjective reason may be that patients avoided going to hospital during this time. Currently, the treatment for patients allergic to inhaled allergens who are not infected with COVID-19 is controversial. ARIA-EAACI ${ }^{33}$ recommends the continuation of SCIT, when infection prevention and control measures are strictly followed at the hospital according to the request of $\mathrm{WHO}^{34,35}$. On the contrary, American Academy of Allergy, Asthma \& Immunology (AAAAI) ${ }^{9}$ suggests that 
it is possible to delay SCIT injections to an interval of once every 2 weeks in the build-up phase, and once every 6 weeks in the maintenance phase (which matched our study design), or even to suspend treatment until social quarantine is cancelled. These diverse viewpoints inspired us to investigate whether there was a difference in clinical presentation between patients receiving SCIT on schedule, and those with a treatment delay. Our results showed that clinical symptoms, medication use, and quality of life were all within the normal range for SCIT patients. In addition, we found no significant difference between scheduled SCIT and delayed SCIT patients at the 1 year follow up period. The result demonstrated again the long-term efficacy of SCIT in patients with AR, even with a delay in therapy during the COVID-19 pandemic. This information may encourage patients who have had to delay SCIT to continue their treatment once the necessary social quarantine is over, to recover their allergen-specific immune tolerance and modify the progression of the disease.

Psychological disorder was also a notable element among patients undergoing SCIT during the COVID-19 pandemic. In a report from China, up to $35.1 \%, 20.1 \%, 18.2 \%$ of people showed depressive symptoms, the symptoms of anxiety disorders, and a change in sleep quality during the COVID-19 pandemic, respectively ${ }^{36}$. In our study, the depressive symptoms in both scheduled and delayed SCIT patients at V0 was significantly higher than at V1, and exceeded the normal range at both time points. Depressed patients reported an increase in symptoms, reduced quality of life, and poor control of symptoms during the SCIT, which was positively correlated with delayed treatment at V0. Our findings may serve to remind allergists/immunologists that the psychological burden on patients undergoing SCIT during the COVID-19 epidemic should be valued with the great attention, particularly with regard to depressive symptoms ${ }^{37}$. The introduction of patient education to relieve depression, should be included as part of the management of SCIT, has previously been suggested $^{38}$.

Further developing the application of patient education and telemedicine is an important part of patient care during the unique circumstances brought by the COVID-19 pandemic ${ }^{39,40}$. Allergists/immunologists have been urged to respond to this need by extending the long-standing trust developed through years of faceto-face encounters into online resources ${ }^{41}$. To assist patients with scheduled SCIT, allergists/immunologists are recommended to post information on their web site and social media channels regarding frequently asked questions surrounding the COVID-19 outbreak, the changes to their SCIT practice setting, the differences between acute AR and asthma symptoms, as well as indications for COVID-19 testing. All of this can lower the psychological distress for patients before they seek help at hospitals or $\operatorname{clinics}^{42,43}$. The patients with delayed SCIT are recommended to continue with follow-up visits online, and when possible, home delivery and digital medicine services can be used to obtain scheduled questionnaires and monitor drug administration $^{44,45}$. Local community organizations and health services can also be utilized to assist patients who are treated at home, and who may need support services to ensure optimal care ${ }^{46-49}$. This unique period during the COVID-19 pandemic brings not only challenges, but also the opportunities to update and improve the management of SCIT.

Our RWE study provides valuable information on physical and mental manifestations among patients undergoing delayed SCIT in a real-world setting, which is essential to the evidence base required for treatment practices $^{50}$. Due to the limited design of the current study we are not able to make conclusions regarding the underlying mechanism of delayed SCIT immune responses. To investigate this further, immunopathological features such as allergen-specific immunoglobulin E ( $\mathrm{IgE}$ ) and immunoglobulin G4 antibodies, may be helpful.

In conclusion, we were able to confirm the long-term efficacy of SCIT in patients with AR, even in patients with delayed treatment due to the COVID-19 pandemic. In addition, comorbid mild depressive symptoms, which were more common in patients with not fully controlled SCIT, may require more attention.

\section{Reference}

1. Larsen JN, Broge L, Jacobi H. Allergy immunotherapy: the future of allergy treatment. Drug discovery today. 2016;21(1):26-37. 
2. Rapiejko P, Jurkiewicz D, Pietruszewska W, Zielnik-Jurkiewicz B, Woroń J, Lipiec A. Treatment strategy of allergic rhinitis in the face of modern world threats. Otolaryngologia polska $=$ The Polish otolaryngology. $2018 ; 72(2): 1-12$.

3. Tie K, Miller C, Zanation AM, Ebert CS, Jr. Subcutaneous Versus Sublingual Immunotherapy for Adults with Allergic Rhinitis: A Systematic Review with Meta-Analyses. The Laryngoscope. 2021.

4. Seidman MD, Gurgel RK, Lin SY, et al. Clinical practice guideline: Allergic rhinitis. Otolaryngologyhead and neck surgery : official journal of American Academy of Otolaryngology-Head and Neck Surgery. 2015;152(1 Suppl):S1-43.

5. Bousquet J, Lockey R, Malling HJ. Allergen immunotherapy: therapeutic vaccines for allergic diseases. A WHO position paper. The Journal of allergy and clinical immunology. 1998;102(4 Pt 1):558-562.

6. Layhadi JA, Eguiluz-Gracia I, Shamji MH. Role of IL-35 in sublingual allergen immunotherapy. Current opinion in allergy and clinical immunology. 2019;19(1):12-17.

7. Kiel MA, Röder E, Gerth van Wijk R, Al MJ, Hop WC, Rutten-van Mölken MP. Real-life compliance and persistence among users of subcutaneous and sublingual allergen immunotherapy. The Journal of allergy and clinical immunology. 2013;132(2):353-360.e352.

8. Chhiba KD, Patel GB, Vu THT, et al. Prevalence and characterization of asthma in hospitalized and nonhospitalized patients with COVID-19. The Journal of allergy and clinical immunology. 2020;146(2):307314.e304.

9. Shaker MS, Oppenheimer J, Grayson M, et al. COVID-19: Pandemic Contingency Planning for the Allergy and Immunology Clinic. The journal of allergy and clinical immunology In practice. 2020;8(5):14771488.e1475.

10. Pfaar O, Demoly P, Gerth van Wijk R, et al. Recommendations for the standardization of clinical outcomes used in allergen immunotherapy trials for allergic rhinoconjunctivitis: an EAACI Position Paper. Allergy. 2014;69(7):854-867.

11. Canonica GW, Baena-Cagnani CE, Bousquet J, et al. Recommendations for standardization of clinical trials with Allergen Specific Immunotherapy for respiratory allergy. A statement of a World Allergy Organization (WAO) taskforce. Allergy. 2007;62(3):317-324.

12. Brożek JL, Bousquet J, Agache I, et al. Allergic Rhinitis and its Impact on Asthma (ARIA) guidelines2016 revision. The Journal of allergy and clinical immunology. 2017;140(4):950-958.

13. Bousquet PJ, Combescure C, Neukirch F, et al. Visual analog scales can assess the severity of rhinitis graded according to ARIA guidelines. Allergy. 2007;62(4):367-372.

14. Bousquet PJ, Combescure C, Klossek JM, Daurès JP, Bousquet J. Change in visual analog scale score in a pragmatic randomized cluster trial of allergic rhinitis. The Journal of allergy and clinical immunology. $2009 ; 123(6): 1349-1354$.

15. Poole CD, Bannister CA, Andreasen JN, Andersen JS, Currie CJ. Estimation of health-related utility (EQ-5D index) in subjects with seasonal allergic rhinoconjunctivitis to evaluate health gain associated with sublingual grass allergen immunotherapy. Health and quality of life outcomes. 2014;12:99.

16. Calderón MA, Casale TB, Demoly P. Validation of Patient-Reported Outcomes for Clinical Trials in Allergic Rhinitis: A Systematic Review. The journal of allergy and clinical immunology In practice. 2019;7(5):14501461.e1456.

17. Clark J, Schall R. Assessment of combined symptom and medication scores for rhinoconjunctivitis immunotherapy clinical trials. Allergy. 2007;62(9):1023-1028. 
18. Juniper EF, Ståhl E, Doty RL, Simons FE, Allen DB, Howarth PH. Clinical outcomes and adverse effect monitoring in allergic rhinitis. The Journal of allergy and clinical immunology. 2005;115(3 Suppl 1):S390-413.

19. Juniper EF, Guyatt GH. Development and testing of a new measure of health status for clinical trials in rhinoconjunctivitis. Clinical and experimental allergy : journal of the British Society for Allergy and Clinical Immunology. 1991;21(1):77-83.

20. Kang Y, Liu S, Yang L, et al. Testing the Bidirectional Associations of Mobile Phone Addiction Behaviors With Mental Distress, Sleep Disturbances, and Sleep Patterns: A One-Year Prospective Study Among Chinese College Students. Frontiers in psychiatry. 2020;11:634.

21. Yuan L, Pan B, Wang W, Wang L, Zhang X, Gao Y. Prevalence and predictors of anxiety and depressive symptoms among patients diagnosed with oral cancer in China: a cross-sectional study. BMC psychiatry. 2020;20(1):394.

22. Liu P, Zeng F, Zhou G, et al. Alterations of the default mode network in functional dyspepsia patients: a resting-state fmri study. Neurogastroenterology and motility : the official journal of the European Gastrointestinal Motility Society. 2013;25(6):e382-388.

23. Peng M, Mo B, Liu Y, et al. Prevalence, risk factors and clinical correlates of depression in quarantined population during the COVID-19 outbreak. Journal of affective disorders. 2020;275:119-124.

24. Wu K, Wei X. Analysis of Psychological and Sleep Status and Exercise Rehabilitation of Front-Line Clinical Staff in the Fight Against COVID-19 in China. Medical science monitor basic research. 2020;26:e924085.

25. Cai X, Hu X, Ekumi IO, et al. Psychological Distress and Its Correlates Among COVID-19 Survivors During Early Convalescence Across Age Groups. The American journal of geriatric psychiatry : official journal of the American Association for Geriatric Psychiatry. 2020;28(10):1030-1039.

26. Zhang T, Yu GD, Gu P, Tang Q, Jin Y, He XC. [Evaluation and analysis of anxiety, depression and quality of life in vasomotor rhinitis]. Zhonghua er bi yan hou tou jing wai ke za zhi $=$ Chinese journal of otorhinolaryngology head and neck surgery. 2020;55(8):769-773.

27. Liu S, Wu R, Li L, et al. The prevalence of anxiety and depression in Chinese asthma patients. PloS one. 2014;9(7):e103014.

28. Hao T, Youxiang M, Xiuyong D, Ruxiang Z, Jiao X. [Prevalence and psychopathological characteristics of anxiety and depression in patients with chronic rhinosinusitis before endoscopic sinus surgery]. Zhonghua er bi yan hou tou jing wai ke za zhi = Chinese journal of otorhinolaryngology head and neck surgery. 2015;50(3):210-214.

29. Li Y, Zhang H, Wang Y. Tai Chi Ameliorates Coronary Heart Disease by Affecting Serum Levels of miR-24 and miR-155. Frontiers in physiology. 2019;10:587.

30. Wang T, Li S, Jiang G, et al. Regional homogeneity changes in patients with primary insomnia. European radiology. 2016;26(5):1292-1300.

31. Guo Z, Tang HY, Li H, et al. The benefits of psychosocial interventions for cancer patients undergoing radiotherapy. Health and quality of life outcomes. 2013;11:121.

32. Pfaar O, Agache I, Bonini M, et al. COVID-19 pandemic and allergen immunotherapy - an EAACI survey. Allergy. 2021;10(2):111.

33. Klimek L, Jutel M, Akdis C, et al. Handling of allergen immunotherapy in the COVID-19 pandemic: An ARIA-EAACI statement. Allergy. 2020;75(7):1546-1554.

34. WH. O. Infection prevention and control of epidemic-and pandemic prone acute respiratory infections in health care. 
35. ECfDPa. C. Guidance for wearing and removing personal protective equipment in healthcare settings for the care of patients with suspected or confirmed COVID-19.

36. Lei L, Huang X, Zhang S, Yang J, Yang L, Xu M. Comparison of Prevalence and Associated Factors of Anxiety and Depression Among People Affected by versus People Unaffected by Quarantine During the COVID-19 Epidemic in Southwestern China. Medical science monitor : international medical journal of experimental and clinical research. 2020;26:e924609.

37. Pfaar O, Klimek L, Jutel M, et al. COVID-19 pandemic: Practical considerations on the organization of an allergy clinic - an EAACI/ARIA Position Paper. Allergy. 2020.

38. Imai R, Hori $\mathrm{H}$, Itoh $\mathrm{M}$, et al. Inflammatory markers and their possible effects on cognitive function in women with posttraumatic stress disorder. Journal of psychiatric research. 2018;102:192-200.

39. Portnoy J, Waller M, Elliott T. Telemedicine in the Era of COVID-19. The journal of allergy and clinical immunology In practice. 2020;8(5):1489-1491.

40. Waibel KH, Bickel RA, Brown T. Outcomes From a Regional Synchronous Tele-Allergy Service. The journal of allergy and clinical immunology In practice. 2019;7(3):1017-1021.

41. Shaker MS, Mosnaim G, Oppenheimer J, Stukus D, Abrams EM, Greenhawt M. Health and Economic Outcomes of Home Maintenance Allergen Immunotherapy in Select Patients with High Health Literacy during the COVID-19 Pandemic: A Cost-Effectiveness Analysis During Exceptional Times. The journal of allergy and clinical immunology In practice. 2020;8(7):2310-2321.e2314.

42. Malipiero G, Paoletti G, Puggioni F, et al. An academic allergy unit during COVID-19 pandemic in Italy. The Journal of allergy and clinical immunology. 2020;146(1):227.

43. Malipiero G, Heffler E, Pelaia C, et al. Allergy clinics in times of the SARS-CoV-2 pandemic: an integrated model. Clinical and translational allergy. 2020;10:23.

44. Codispoti CD, Bandi S, Moy JN, Mahdavinia M. Running a virtual allergy division and training program in the time of COVID-19 pandemic. The Journal of allergy and clinical immunology. 2020;145(5):1357-1359.

45. Matricardi PM, Dramburg S, Alvarez-Perea A, et al. The role of mobile health technologies in allergy care: An EAACI position paper. Allergy. 2020;75(2):259-272.

46. Abi Fadel F, Al-Jaghbeer M, Kumar S, et al. The impact of the state of Ohio stay-at-home order on nonCOVID-19 intensive care unit admissions and outcomes. Anaesthesiology intensive therapy. 2020;52(3):249252.

47. Klimek L, Pfaar O, Worm M, et al. Allergen immunotherapy in the current COVID-19 pandemic: A position paper of AeDA, ARIA, EAACI, DGAKI and GPA: Position paper of the German ARIA Group(A) in cooperation with the Austrian ARIA Group(B), the Swiss ARIA Group(C), German Society for Applied Allergology (AEDA)(D), German Society for Allergology and Clinical Immunology (DGAKI)(E), Society for Pediatric Allergology (GPA)(F) in cooperation with AG Clinical Immunology, Allergology and Environmental Medicine of the DGHNO-KHC $(\mathrm{G})$ and the European Academy of Allergy and Clinical Immunology $($ EAACI $)(H)$. Allergologie select. 2020;4:44-52.

48. Torlinski T. Initial hospital preparation and response to fight the COVID-19 pandemic, based on the British university hospital experience. Anaesthesiology intensive therapy. 2020;52(3):256-258.

49. Zieliński M, Wróblewski P, Kozielski J. Proposed practice guidelines for burn centres in the context of the SARS-CoV-2 pandemic. Anaesthesiology intensive therapy. 2020;52(3):245-248.

50. Jutel M, Brüggenjürgen B, Richter H, Vogelberg C. Real-world evidence of subcutaneous allergoid immunotherapy in house dust mite-induced allergic rhinitis and asthma. Allergy. 2020;75(8):2050-2058.

Table 1. Clinical characteristics of patients with SCIT scheduled and delayed. 


\begin{tabular}{|c|c|c|c|}
\hline & $\begin{array}{l}\text { Patients with SCIT } \\
\text { scheduled }(\mathrm{n}=249)\end{array}$ & $\begin{array}{l}\text { Patients with SCIT } \\
\text { delayed }(\mathrm{n}=394)\end{array}$ & $\mathrm{P}$ value \\
\hline Age $($ mean \pm SD $)$ & $13 \pm 11.34$ & $12 \pm 11.16$ & ns \\
\hline Gender & & & ns \\
\hline Male & 163 & 249 & \\
\hline Female & 86 & 145 & \\
\hline Treatment phase & & & $0.0057^{*}$ \\
\hline Build up phase ${ }^{1}$ & 54 & 53 & \\
\hline Maintenance phase $^{2}$ & 195 & 296 & \\
\hline Diagnosis & & & $0.0001^{*}$ \\
\hline AR only & 246 & 363 & \\
\hline $\mathrm{AR}+$ Asthma & 1 & 31 & \\
\hline $\mathrm{AR}+$ Asthma $+\mathrm{AC}$ & 1 & 0 & \\
\hline $\mathrm{AR}+\mathrm{IBS}$ & 1 & 0 & \\
\hline $\begin{array}{l}\text { Adverse } \\
\text { reactions(V0/V1, \%) }\end{array}$ & & & ns \\
\hline $\begin{array}{l}\text { Immediate local } \\
\text { reactions }\end{array}$ & $0 / 1.9 \%$ & $1.0 \% / 1.9 \%$ & \\
\hline $\begin{array}{l}\text { Immediate systemic } \\
\text { reactions }\end{array}$ & $0 / 1.0 \%$ & $0.3 \% / 0.9 \%$ & \\
\hline Delayed local reactions & $0.4 \% / 1.0 \%$ & $1.8 \% / 2.8 \%$ & \\
\hline $\begin{array}{l}\text { Delayed systemic } \\
\text { reactions }\end{array}$ & $0 / 0$ & $0 / 0.5 \%$ & \\
\hline Convid-19 infection & 0 & 0 & \\
\hline
\end{tabular}

Data are provided as n, unless indicated otherwise. SCIT: subcutaneous immunotherapy; SD: standard deviation; AR: Allergic rhinitis; AC: Allergic conjunctivities; IBS: irritable bowel syndrome; V0: patients at the first visit; V1: patients at 1-year follow up; COVID-19: coronavirus disease 2019.

${ }^{1}$ AIT was given by increased dose every 1-2 weeks until the maximum tolerated dose of the individual was reached.

${ }^{2}$ The maximum tolerated dose of the patients was given subcutaneously every 4-6 weeks for 2 years.

${ }^{*} \mathrm{P}$ value of $<0.05$ was considered as statistically significant.

Table 2. Clinical manifestations in detail of patients with SCIT scheduled and delayed.

\begin{tabular}{|c|c|c|c|c|c|c|}
\hline & $\begin{array}{l}\text { V0 } \\
\text { SCIT } \\
\text { scheduled }\end{array}$ & $\begin{array}{l}\text { V0 } \\
\text { SCIT } \\
\text { delayed }\end{array}$ & $\begin{array}{l}\text { V0 } \\
\mathrm{P} \text { value }\end{array}$ & $\begin{array}{l}\text { V1 } \\
\text { SCIT } \\
\text { scheduled }\end{array}$ & $\begin{array}{l}\text { V1 } \\
\text { SCIT } \\
\text { delayed }\end{array}$ & $\begin{array}{l}\mathrm{V} 1 \\
\mathrm{P} \text { value }\end{array}$ \\
\hline \multicolumn{7}{|l|}{ VAS } \\
\hline Never & $9(4.27 \%)$ & $50(14.79 \%)$ & $0.0001^{*}$ & $10(9.62 \%)$ & $20(9.42 \%)$ & ns \\
\hline Mild & $\begin{array}{l}125 \\
(59.24 \%)\end{array}$ & $\begin{array}{l}195 \\
(57.69 \%)\end{array}$ & ns & $73(70.19 \%)$ & $\begin{array}{l}152 \\
(71.71 \%)\end{array}$ & ns \\
\hline Moderate & $64(30.33 \%)$ & $71(21.01 \%)$ & $0.0136^{*}$ & $17(16.35 \%)$ & $39(18.40 \%)$ & $\mathrm{ns}$ \\
\hline $\begin{array}{l}\text { Severe } \\
\text { dSS }\end{array}$ & $13(6.16 \%)$ & $22(6.51 \%)$ & ns & $4(3.84 \%)$ & $1(0.47 \%)$ & $0.0424^{*}$ \\
\hline $\begin{array}{l}\text { Nasal } \\
\text { symptoms }\end{array}$ & $213(85.54 \%)$ & $319(83.51 \%)$ & ns & $81(77.14 \%)$ & $183(86.73 \%)$ & $0.0231^{*}$ \\
\hline Itchy nose & $\begin{array}{l}147 \\
(59.51 \%)\end{array}$ & $\begin{array}{l}200 \\
(50.76 \%)\end{array}$ & $\mathrm{ns}$ & $42(39.90 \%)$ & $\begin{array}{l}154 \\
(54.46 \%)\end{array}$ & $0.0001^{*}$ \\
\hline
\end{tabular}




\begin{tabular}{|c|c|c|c|c|c|c|}
\hline Sneezing & $\begin{array}{l}187 \\
(75.10 \%)\end{array}$ & $\begin{array}{l}280 \\
(71.98 \%)\end{array}$ & ns & $54(51.30 \%)$ & $\begin{array}{l}154 \\
(71.96 \%)\end{array}$ & $0.0001^{*}$ \\
\hline Runny nose & $152(61.54 \%)$ & $222(57.22 \%)$ & ns & $61(58.10 \%)$ & $123(57.48 \%)$ & ns \\
\hline Blocked nose & $136(55.06 \%)$ & $203(52.18 \%)$ & ns & $53(50.48 \%)$ & $115(53.74 \%)$ & $\mathrm{ns}$ \\
\hline $\begin{array}{l}\text { Conjunctival } \\
\text { symptoms }\end{array}$ & $117(47.56 \%)$ & $173(43.91 \%)$ & ns & $34(32.38 \%)$ & $85(39.91 \%)$ & ns \\
\hline $\begin{array}{l}\text { Itchy/red } \\
\text { eyes }\end{array}$ & $\begin{array}{l}114 \\
(46.15 \%)\end{array}$ & $\begin{array}{l}167 \\
(42.49 \%)\end{array}$ & ns & $33(31.43 \%)$ & $80(37.38 \%)$ & ns \\
\hline $\begin{array}{l}\text { Watery eyes } \\
\text { dMS }\end{array}$ & $71(28.97 \%)$ & $82(21.13 \%)$ & $0.0267^{*}$ & $17(16.19 \%)$ & $35(16.43 \%)$ & ns \\
\hline Never & $202(81.78 \%)$ & $295(75.84 \%)$ & ns & $90(85.71 \%)$ & $183(85.91 \%)$ & ns \\
\hline H1A & $29(11.74 \%)$ & $43(11.05 \%)$ & ns & $9(8.58 \%)$ & $16(7.51 \%)$ & ns \\
\hline $\begin{array}{l}\text { INS } \\
\text { with/without } \\
\text { H1A }\end{array}$ & $10(4.05 \%)$ & $35(9.00 \%)$ & $0.0177^{*}$ & $6(5.71 \%)$ & $9(4.22 \%)$ & $\mathrm{ns}$ \\
\hline $\begin{array}{l}\text { Oral } \\
\text { corticosteroids } \\
\text { with/without } \\
\text { INS, } \\
\text { with/without } \\
\text { H1A } \\
\text { QoL }\end{array}$ & $6(2.43 \%)$ & $16(4.11 \%)$ & ns & 0 & $5(2.36 \%)$ & - \\
\hline $\begin{array}{l}\text { Activity } \\
\text { problems }\end{array}$ & $194(78.54 \%)$ & $226(57.80 \%)$ & $0.0001^{*}$ & $71(67.62 \%)$ & $124(57.94 \%)$ & ns \\
\hline Sleep problems & $144(57.83 \%)$ & $201(51.40 \%)$ & ns & $47(44.76 \%)$ & $128(59.81 \%)$ & $0.0097^{*}$ \\
\hline $\begin{array}{l}\text { Generalized } \\
\text { symptoms }\end{array}$ & $162(65.59 \%)$ & $213(55.22 \%)$ & $0.0093^{*}$ & $51(48.57 \%)$ & $133(62.15 \%)$ & $0.0185^{*}$ \\
\hline $\begin{array}{l}\text { Practical } \\
\text { problems }\end{array}$ & $179(72.47 \%)$ & $302(76.84 \%)$ & ns & $31(67.39 \%)$ & $169(78.97 \%)$ & $0.0001^{*}$ \\
\hline $\begin{array}{l}\text { Nasal } \\
\text { symptoms }\end{array}$ & $\begin{array}{l}208 \\
(84.21 \%)\end{array}$ & $\begin{array}{l}334 \\
(84.99 \%)\end{array}$ & ns & $77(73.33 \%)$ & $\begin{array}{l}183 \\
(85.51 \%)\end{array}$ & $0.0063^{*}$ \\
\hline $\begin{array}{l}\text { Ocular } \\
\text { symptoms }\end{array}$ & $137(55.46 \%)$ & $218(55.75 \%)$ & ns & $41(39.05 \%)$ & $127(59.35 \%)$ & $0.0005^{*}$ \\
\hline $\begin{array}{l}\text { Emotional } \\
\text { problems }\end{array}$ & $\begin{array}{l}122 \\
(49.39 \%)\end{array}$ & $\begin{array}{l}164 \\
(41.94 \%)\end{array}$ & ns & $35(33.33 \%)$ & $\begin{array}{l}102 \\
(47.89 \%)\end{array}$ & $0.0137^{*}$ \\
\hline
\end{tabular}

Date were $\mathrm{n}$ or (percentage). V0: patients at the first visit; V1: patients at 1-year follow up; SCIT: subcutaneous immunotherapy; VAS: visual analog scale; dSS: daily symptom score; dMS: daily medication score; H1A: oral and/or topical (eyes or nose) nonsedative H1 antihistamines; INS: intranasal corticosteroids; QoL: quality of Life.

${ }^{*} \mathrm{P}$ value of $<0.05$ was considered as statistically significant.

${ }^{1}$ QoL evluation by quality of life questionnaire (RQLQ).

Figure legends

Figure 1. Study flow chart.

SCIT: subcutaneous immunotherapy; V0: patients at the first visit; V1: patients at 1-year follow up; VAS: visual analogue scale; dSS: daily symptom score; dMS: daily medication score; CSMS: combined symptom and medication scores; QoL: quality of life; SAS: self-rating anxiety scale; SDS: self-rating depression scale. 
Figure 2. Clinical manifestations in patients with scheduled and delayed SCIT.

(A-E), Mean values with 25 th and 75 th percentiles are indicated by scale bar. ${ }^{* * * *} \mathrm{P}<0.0001$, ${ }^{* * *} \mathrm{P}<$ $0.001,{ }^{*} \mathrm{P}<0.01,{ }^{*} \mathrm{P}<0.05$.

SCIT: subcutaneous immunotherapy; VAS: visual analogue scale; dSS: daily symptom score; dMS: daily medication score; CSMS: combined symptom and medication scores; QoL: quality of life; V0: patients at the first visit; V1: patients at 1-year follow up.

Figure 3. Emotional evaluation of different diseases and SCIT among patients in this study.

(A-B), Compared SAS and SDS of delayed SCIT group at V1, to delayed SCIT group and V0, scheduled SCIT group at V0 and V1 in this study and different diseases. Mean values with SD are indicated by scale bar.

(C), Proportion of SDS in patients with scheduled or delayed scheduled or delayed at V0 and V1. Mean values with 25 th and 75 th percentiles are indicated by scale bar.

(D), Correlation of SDS with time interval in the delayed SCIT group at V0.

**** $\mathrm{P}<0.0001,{ }^{* * *} \mathrm{P}<0.001,{ }^{* *} \mathrm{P}<0.01,{ }^{*} \mathrm{P}<0.05$.

SCIT: subcutaneous immunotherapy; SAS: self-rating anxiety scale; SDS: self-rating depression scale; COVID-19: coronavirus disease 2019; AR: allergic rhinitis; CRS: chronic rhinosinusitis; SD: standard deviation; RT:radiotherapy; V0: patients at the first visit; V1: patients at 1-year follow up.

Figure 4. Compared clinical manifestations and control to depressive symptoms.

(A-E), Compared VAS, dSS, dMS, CSMS, and QoL of SCIT patients in non-depressed group to depressed group at V0 and V1. Mean values with 25 th and 75 th percentiles are indicated by scale bar. $* * * * \mathrm{P}<0.0001$, ${ }^{* * *} \mathrm{P}<0.001,{ }^{* *} \mathrm{P}<0.01,{ }^{*} \mathrm{P}<0.05$.

(F), Compared clinical control of SCIT patients to depressive symptom at V0 and V1. Mean values with 25th and 75th percentiles are indicated by scale bar. ${ }^{* * *} \mathrm{P}<0.001,{ }^{* *} \mathrm{P}<0.01,{ }^{*} \mathrm{P}<0.05$.

SCIT: subcutaneous immunotherapy; VAS: visual analogue scale; dSS: daily symptom score; dMS: daily medication score; CSMS: combined symptom and medication scores; QoL: quality of life; SDS: self-rating depression scale; V0: patients at the first visit; V1: patients at 1-year follow up. 